\title{
THE SHARP REMEZ-TYPE INEQUALITY FOR EVEN TRIGONOMETRIC POLYNOMIALS ON THE PERIOD
}

\author{
TAMÁS ERDÉLYI
}

September 7, 2018

Abstract. We prove that

$$
\max _{t \in[-\pi, \pi]}|Q(t)| \leq T_{2 n}(\sec (s / 4))=\frac{1}{2}\left((\sec (s / 4)+\tan (s / 4))^{2 n}+(\sec (s / 4)-\tan (s / 4))^{2 n}\right)
$$

for every even trigonometric polynomial $Q$ of degree at most $n$ with complex coefficients satisfying

$$
m(\{t \in[-\pi, \pi]:|Q(t)| \leq 1\}) \geq 2 \pi-s, \quad s \in(0,2 \pi),
$$

where $m(A)$ denotes the Lebesgue measure of a measurable set $A \subset \mathbb{R}$ and $T_{2 n}$ is the Chebysev polynomial of degree $2 n$ on $[-1,1]$ defined by $T_{2 n}(\cos t)=\cos (2 n t)$ for $t \in \mathbb{R}$. This inequality is sharp. We also prove that

$$
\max _{t \in[-\pi, \pi]}|Q(t)| \leq T_{2 n}(\sec (s / 2))=\frac{1}{2}\left((\sec (s / 2)+\tan (s / 2))^{2 n}+(\sec (s / 2)-\tan (s / 2))^{2 n}\right)
$$

for every trigonometric polynomial $Q$ of degree at most $n$ with complex coefficients satisfying

$$
m(\{t \in[-\pi, \pi]:|Q(t)| \leq 1\}) \geq 2 \pi-s, \quad s \in(0, \pi) .
$$

\section{FOREWORD}

I started my Ph.D. program in June, 1987, at The Ohio State University, as a student of Paul Nevai. In August, 1987, Paul Nevai moved from Columbus, Ohio, to Columbia, South Carolina, to spend the school year 1987-88 at the University of South Carolina, and each of his students followed him. This is how I met Mr. (Yingkang) Hu, who was a student of Ron DeVore at that time. It was a somewhat short but very exciting time for me at the University of South Carolina. Not only had I passed each of my necessary exams in my Ph.D. program but I had the possibility to learn from and interact with some of the very best researchers in approximation theory. My office neighbors were Paul Nevai, Ron DeVore, and George Lorenz. George Lorentz happened to be Ron DeVore's

Key words and phrases. trigonometric polynomials, Remez-type inequalities, geometry of polynomials. 2010 Mathematics Subject Classifications. 11C08, 41A17, 26C10, 30C15 
office neighbor on one hand and my office neighbor on the other hand to finalize the book entitled "Constructive Approximation" he was writing jointly with Ron DeVore. It was a privilege for me that the authors of this fundamental book in approximation theory asked me to read some sections of their book in preparation and took my remarks seriously. I was also lucky to have talented, motivated, and helpful class-mates such as Yingkang $\mathrm{Hu}$, George Kyriazis, Jiaxiang (John) Zhang, and Shing-Whu Jha around. During the school year 1987-88 Yingkang was a good friend of mine. We spent much time together as classmates as well as friends. I liked to eat Chinese food in the company of Mr. Hu, Jiaxiang Zhang, Shing-Whu Jha, and some of my other Chinese friends. We all took Paul Nevai's class on Orthogonal Polynomials. We traveled quite much together in this school year to attend various conferences, but Yinkang, Jiaxiang, and me traveled together as tourists as well in the spring break. In August, 1988, Paul Nevai moved back to Columbus, and I followed him to write my dissertation at The Ohio State University. I defended my Ph.D. dissertation at the University of South Carolina in May, 1989. A central part of my dissertation was a Remez-type inequality for (generalized) algebraic and trigonometric polynomials and their applications in the proofs of various other inequalities for generalized polynomials. I was not able to prove the sharp Remez-type inequality for trigonometric polynomials, but I have managed to prove the sharp upper bound for $|Q(0)|$ for even trigonometric polynomials $Q$ satisfying

$$
m\{t \in(0,2 \pi]:|Q(t)| \leq 1\}) \geq 2 \pi-s, \quad s \in(0,2 \pi)
$$

where $m(A)$ denotes the Lebesgue measure of a set $A \subset \mathbb{R}$. After some 30 years I have revisited the topic of my dissertation. While the sharp Remez-type inequality for trigonometric polynomials remains open, I am glad to prove the sharp Remez-type inequality at least for even trigonometric polynomials in this volume dedicated to the memory of Dr. Hu. After defending my thesis I met Yingkang very rarely, but we were keeping in touch. In March, 2002, Yingkang visited us at Texas A\&M University, and gave a talk in our Center of Approximation Theory Seminar with the title "Equivalence of Moduli of Smoothness", a topic on which he published two papers in Journal of Approximation Theory. During his visit we watched the Academy Award Ceremony in my apartment as he was very much interested in some of the latest movies. This may have been the last time I saw him and I was very sad to learn about his death in March, 2016.

\section{INTRODUCTION}

Let $\mathcal{T}_{n}$ denote the set of all real trigonometric polynomials of degree at most $n$. Let $\mathcal{T}_{n}^{c}$ denote the set of all complex trigonometric polynomials of degree at most $n$. Let $K:=\mathbb{R}(\bmod 2 \pi)$. Let $m(A)$ denote the Lebesgue measure of a measurable set $A \subset \mathbb{R}$. For $s \in(0,2 \pi)$ let

$$
\mathcal{T}_{n}(s):=\left\{Q \in \mathcal{T}_{n}: m(\{t \in K:|Q(t)| \leq 1\}) \geq 2 \pi-s\right\}
$$

and

$$
\mathcal{T}_{n}^{c}(s):=\left\{Q \in \mathcal{T}_{n}^{c}: m(\{t \in K:|Q(t)| \leq 1\}) \geq 2 \pi-s\right\}
$$


Let $\mathcal{P}_{n}$ denote the set of all algebraic polynomials of degree at most $n$ with real coefficients. Let $\mathcal{P}_{n}^{c}$ denote the set of all algebraic polynomials of degree at most $n$ with complex coefficients. Let $T_{n}$ be the Chebysev polynomial of degree $n$ on $[-1,1]$ defined by $T_{n}(\cos t)=\cos (n t)$ for $t \in K$. For real numbers $a<b$ and a Lebesgue measurable set $A \subset[a, b]$ let

$$
\mu_{[a, b]}(A):=\int_{A} \frac{(b-a) / 2}{\sqrt{((b-a) / 2)^{2}-(x-(a+b) / 2)^{2}}} d x .
$$

The classical Remez inequality states that if $P \in \mathcal{P}_{n}, s \in(0,2)$, and

$$
m(\{x \in[-1,1]:|P(x)| \leq 1\}) \geq 2-s,
$$

then

$$
\max _{x \in[-1,1]}|P(x)| \leq T_{n}\left(\frac{2+s}{2-s}\right)
$$

where $T_{n}$ defined by

$$
T_{n}(x):=\cos (n \arccos x), \quad x \in[-1,1]
$$

is the Chebyshev polynomial of degree $n$. This inequality is sharp and

$$
T_{n}\left(\frac{2+s}{2-s}\right) \leq \exp \left(\min \left\{5 n s^{1 / 2}, 2 n^{2} s\right\}\right), \quad s \in(0,1]
$$

Remez-type inequalities for various classes of functions have been studied by several authors, and they have turned out to be applicable and connected to various problems in approximation theory. See [1]-[34], for example.

In [16] we proved that

$$
|Q(0)| \leq T_{2 n}(\sec (s / 4))
$$

for every even $Q \in \mathcal{T}_{n}^{c}(s)$ and $s \in(0,2 \pi)$. However, 0 is a special point in the study of even trigonometric polynomials $Q \in \mathcal{T}_{n}^{c}(s)$, so the question whether or not

$$
\max _{t \in K}|Q(t)| \leq T_{2 n}(\sec (s / 4))
$$

holds at least for all even trigonometric polynomials $Q \in \mathcal{T}_{n}^{c}(s)$ remained open, while it was speculated that (1.2) may hold for all $Q \in \mathcal{T}_{n}^{c}(s)$. In this paper we show that (1.2) holds for all even $Q \in \mathcal{T}_{n}^{c}(s)$, while it remains open whether or not (1.2) holds for all $Q \in \mathcal{T}_{n}^{c}(s)$.

\section{NeW Results}

Our first result is a sharp Remez-type inequality for even trigonometric polynomials with complex coefficients. 
Theorem 2.1. We have

$$
\max _{t \in K}|Q(t)| \leq T_{2 n}(\sec (s / 4))=\frac{1}{2}\left((\sec (s / 4)+\tan (s / 4))^{2 n}+(\sec (s / 4)-\tan (s / 4))^{2 n}\right)
$$

for every even $Q \in \mathcal{T}_{n}^{c}(s)$ and $s \in(0,2 \pi)$. Equality holds if and only if

$$
\{t \in K:|Q(t)| \leq 1\}=[s / 2,2 \pi-s / 2]
$$

and $Q \in \mathcal{T}_{n}(s)$ is of the form

$$
Q(t)=T_{2 n}\left(\frac{\cos (t / 2)}{\cos (s / 4)}\right)
$$

Our next result is a Remez-type inequality for odd trigonometric polynomials with complex coefficients.

Theorem 2.2. We have

$$
\max _{t \in K}|Q(t)| \leq \frac{1}{2}\left((\sec (s / 4)+\tan (s / 4))^{2 n}+(\sec (s / 4)-\tan (s / 4))^{2 n}\right)+\frac{1}{\sqrt{2}}
$$

for every odd $Q \in \mathcal{T}_{n}^{c}(s)$ and $s \in(0,2 \pi)$.

Theorem 2.1 implies he following result for all trigonometric polynomials with complex coefficients.

Theorem 2.3. We have

$$
\max _{t \in K}|R(t)| \leq T_{2 n}(\sec (s / 2))=\frac{1}{2}\left((\sec (s / 2)+\tan (s / 2))^{2 n}+(\sec (s / 2)-\tan (s / 2))^{2 n}\right)
$$

for every $R \in \mathcal{T}_{n}^{c}(s)$ and $s \in(0, \pi)$.

Note that Theorem 2.2 was proved in [16] only for all $R \in \mathcal{T}_{n}(s)$ (rather than $R \in \mathcal{T}_{n}^{c}(s)$ ) and $s \in(0, \pi)$. It remains open whether or not Theorem 2.1 can be extended to all $Q \in \mathcal{T}_{n}^{c}(s)$ and $s \in(0,2 \pi)$.

\section{LEMMAS}

Our first lemma is the conclusion (13) of Section 7 in [16]. This deep result plays a central role in the proof of Theorem 2.1.

Lemma 3.1. We have

$$
|U(0)| \leq T_{2 n}(\sec (s / 4))
$$

for every even $U \in \mathcal{T}_{n}(s)$ and $s \in(0,2 \pi)$. Equality holds if and only if

$$
\{t \in K:|U(t)| \leq 1\}=[s / 2,2 \pi-s / 2]
$$

and

$$
U(t)=T_{2 n}\left(\frac{\cos (t / 2)}{\cos (s / 4)}\right) .
$$

Our next lemma extends Lemma 3.1 to all even trigonometric polynomials with complex coefficients. 
Lemma 3.2. We have

$$
|Q(0)| \leq T_{2 n}(\sec (s / 4))
$$

for every even $Q \in \mathcal{T}_{n}^{c}(s)$ and $s \in(0,2 \pi)$. Equality holds if and only if

$$
\{t \in K:|Q(t)| \leq 1\}=[s / 2,2 \pi-s / 2]
$$

and

$$
Q(t)=T_{2 n}\left(\frac{\cos (t / 2)}{\cos (s / 4)}\right)
$$

Proof. Let $Q \in \mathcal{T}_{n}^{c}(s)$. Choose $c \in \mathbb{C}$ with $|c|=1$ so that $c Q(0)$ is real. Define $U \in \mathcal{T}_{n}(s)$ by

$$
U(t):=\operatorname{Re}(c Q(t)), \quad t \in K .
$$

Applying Lemma 3.1 to $U \in \mathcal{T}_{n}(s)$ we obtain

$$
|Q(0)|=|S(0)| \leq T_{n}(\sec (s / 4)),
$$

and equality holds if and only if

$$
\{t \in K:|Q(t)| \leq 1\}=[-\pi+s / 2, \pi-s / 2]
$$

and

$$
Q(t)=T_{2 n}\left(\frac{\cos (t / 2)}{\cos (s / 4)}\right)
$$

Observe that every even $U \in \mathcal{T}_{n}^{c}$ can be written as $U(t)=P(\cos t)$, where $P \in \mathcal{P}_{n}^{c}$. In terms of algebraic polynomials Lemma 3.1 can be formulated as follows.

Lemma 3.3. We have

$$
|P(1)| \leq T_{2 n}(\sec (s / 4))
$$

for every $P \in \mathcal{P}_{n}^{c}$ such that

$$
\mu_{[-1,1]}(\{x \in[-1,1]:|P(x)| \leq 1\}) \geq \pi-s / 2 .
$$

Equality holds if and only if

$$
\{x \in[-1,1]:|P(x)| \leq 1\}=[-1, \cos (s / 2)]
$$

and

$$
P(\cos t)=T_{2 n}\left(\frac{\cos (t / 2)}{\cos (s / 4)}\right)
$$

Associated with $P \in \mathcal{P}_{n}^{c}$ let $\widetilde{P} \in \mathcal{P}_{n}^{c}$ be defined by $\widetilde{P}(x)=P(-x)$. Observing that

$$
\mu_{[-1,1]}(\{x \in[-1,1]:|P(x)| \leq 1\})=\mu_{[-1,1]}(\{x \in[-1,1]:|\widetilde{P}(x)| \leq 1\})
$$

we obtain the following. 
Lemma 3.3*. We have

$$
|P(-1)| \leq T_{2 n}(\sec (s / 4))
$$

for every $P \in \mathcal{P}_{n}^{c}$ such that

$$
\mu_{[-1,1]}(\{x \in[-1,1]:|P(x)| \leq 1\}) \geq \pi-s / 2 .
$$

Equality holds if and only if

$$
\{x \in[-1,1]:|P(x)| \leq 1\}=[-\cos (s / 2), 1]
$$

and

$$
P(-\cos t)=T_{2 n}\left(\frac{\cos (t / 2)}{\cos (s / 4)}\right) .
$$

We need to transform the above two lemmas linearly from the interval $[-1,1]$ to the interval $[a, b]$.

Lemma 3.4. We have

$$
|P(b)| \leq T_{2 n}(\sec (s / 4))
$$

for every $P \in \mathcal{P}_{n}^{c}$ such that

$$
\mu_{[a, b]}(\{x \in[a, b]:|P(x)| \leq 1\}) \geq \frac{b-a}{2}(\pi-s / 2) .
$$

Equality holds if and only if

$$
\{x \in[a, b]:|P(x)| \leq 1\}=[a,(a+b) / 2+((b-a) / 2) \cos (s / 2)]
$$

and

$$
P(((b-a) / 2) \cos t+(a+b) / 2)=T_{2 n}\left(\frac{\cos (t / 2)}{\cos (s / 4)}\right) .
$$

Lemma 3.4*. We have

$$
|P(a)| \leq T_{2 n}(\sec (s / 4))
$$

for every $P \in \mathcal{P}_{n}^{c}$ such that

$$
\mu_{[a, b]}(\{x \in[a, b]:|P(x)| \leq 1\}) \geq \frac{b-a}{2}(\pi-s / 2) .
$$

Equality holds if and only if

$$
\{x \in[a, b]:|P(x)| \leq 1\}=[(a+b) / 2-((b-a) / 2) \cos (s / 2), b]
$$

and

$$
P((-(b-a) / 2) \cos t+(a+b) / 2)=T_{2 n}\left(\frac{\cos (t / 2)}{\cos (s / 4)}\right) .
$$

Observing that $P \in \mathcal{P}_{n}^{c}$ implies $\widetilde{P} \in \mathcal{P}_{n}^{c}$, where $\widetilde{P}(x)=P(-x)$ we obtain the following. 
Lemma 3.5. We have

$$
\mu_{[1-2 r, 1]}(A)>r^{1 / 2} \mu_{[-1,1]}(A)
$$

for every $r \in(0,1)$ and for every Lebesgue measurable set $A \subset[1-2 r, 1]$ with $m(A)>0$. Proof. Let $r \in(0,1)$ and let $A \subset[1-2 r, 1]$ be a Lebesgue measurable set with $m(A)>0$. We have

$$
\begin{aligned}
\frac{\mu_{[1-2 r, 1]}(A)}{\mu_{[-1,1]}(A)} & =\frac{\int_{A} \frac{r}{\sqrt{r^{2}-(x-(1-r))^{2}}} d x}{\int_{A} \frac{1}{\sqrt{\left(1-x^{2}\right.}} d x}=\frac{\int_{A} \frac{r}{\sqrt{\left(1-x^{2}\right)}} \frac{\sqrt{\left(1-x^{2}\right.}}{\sqrt{r^{2}-(x-(1-r))^{2}}} d x}{\int_{A} \frac{1}{\sqrt{1-x^{2}}} d x} \\
& >\frac{\int_{A} \frac{r}{\sqrt{1-x^{2}}} d x \min _{y \in[1-2 r, 1]} \frac{\sqrt{1-y^{2}}}{\sqrt{r^{2}-(y-(1-r))^{2}}}}{\int_{A} \frac{1}{\sqrt{1-x^{2}}} d x} \\
& \geq r r^{-1 / 2}=r^{1 / 2}
\end{aligned}
$$

as

$$
\min _{y \in[1-2 r, 1]} \frac{\sqrt{1-y^{2}}}{\sqrt{r^{2}-(y-(1-r))^{2}}}=\min _{y \in[1-2 r, 1]} \frac{\sqrt{1+y}}{\sqrt{y-(1-2 r)}}=\frac{\sqrt{2}}{\sqrt{2 r}}=r^{-1 / 2} .
$$

Lemma 3.6. We have

$$
\mu_{[-1,1-2 r]}(A)>(1-r)^{1 / 2} \mu_{[-1,1]}(A)
$$

for every $r \in(0,1)$ and for every Lebesgue measurable set $A \subset[-1,-1+2 r]$ with $m(A)>0$. Proof. Let $r \in(0,1)$ and let $A \subset[-1,1-2 r]$ be a Lebesgue measurable set with $m(A)>0$.. Applying Lemma 3.5 with $A$ replaced by $-A:=\{-x: x \in A\} \subset[1-2(1-r), 1]$ and $r \in(0,1)$ replaced by $1-r \in(0,1)$, we get

$$
\mu_{[-1,-1+2 r]}(A)=\mu_{[1-2(1-r), 1]}(-A)>(1-r)^{1 / 2} \mu_{[-1,1]}(-A)=(1-r)^{1 / 2} \mu_{[-1,1]}(A) .
$$

Lemma 3.7. We have

$$
\arccos (1-2 r)>\pi r^{1 / 2} \quad \text { and } \quad \pi-\arccos (1-2 r)>\pi(1-r)^{1 / 2}
$$

for all $r \in(0,1)$.

Proof. The first inequality is equivalent to $\sin t>(2 / \pi) t$ for all $t=(\pi / 2) r^{1 / 2} \in(0, \pi / 2)$. The second inequality follows from the first one as

$$
\pi-\arccos (1-2 r)=\arccos (-1+2 r)=\arccos (1-2(1-r))>\pi(1-r)^{1 / 2}
$$

for all $r \in(0,1)$. 


\section{Proof of Theorem 2.1}

Proof of Theorem 2.1. Let $Q \in \mathcal{T}_{n}^{c}(s)$ be even with $s \in(0,2 \pi)$. Then $Q$ is of the form $Q(t)=P(\cos t)$, where $P \in \mathcal{P}_{n}^{c}$ satisfies

$$
\mu_{[-1,1]}(\{x \in[-1,1]:|P(x)| \leq 1\}) \geq \pi-s / 2 .
$$

We want to prove that

$$
|P(\alpha)| \leq T_{2 n}(\sec (s / 4))
$$

for all $\alpha \in[-1,1]$.

If $\alpha=1$ then (4.2) holds by Lemma 3.3. If $\alpha=-1$ then (4.2) holds by Lemma 3.3*. Assume now that $\alpha=1-2 r \in(-1,1)$, that is, $\alpha=1-2 r$ with $r \in(0,1)$. It follows from (4.1) and the definition of the measure $\mu_{[-1,1]}$ that

$$
m(\{t \in[0, \pi]:|P(\cos t)| \leq 1)\}) \geq \pi-s / 2
$$

and hence we have either

$$
m(\{t \in[0, \arccos (1-2 r)]:|P(\cos t)| \leq 1)\}) \geq \frac{\pi-\arccos (1-2 r)}{\pi}(\pi-s / 2)
$$

or

$$
m(\{t \in[\arccos (1-2 r), \pi]:|P(\cos t)| \leq 1)\}) \geq \frac{\arccos (1-2 r)}{\pi}(\pi-s / 2) .
$$

For the sake of brevity let

$$
A_{1}:=\{x \in[-1,1-2 r]:|P(x)| \leq 1\} \quad \text { and } \quad A_{2}:=\{x \in[1-2 r, 1]:|P(x)| \leq 1\}
$$

By (4.3), (4.4), and (4.5) we have either

$$
\mu_{[-1,1]}\left(A_{1}\right) \geq \frac{\pi-\arccos (1-2 r)}{\pi}(\pi-s / 2)
$$

or

$$
\mu_{[-1,1]}\left(A_{2}\right) \geq \frac{\arccos (1-2 r)}{\pi}(\pi-s / 2)
$$

Suppose that (4.6) holds. Then, combining Lemmas 3.6 and 3.7 we obtain

$$
\begin{aligned}
& \mu_{[-1,1-2 r]}\left(A_{1}\right)>(1-r)^{1 / 2} \mu_{[-1,1]}\left(A_{1}\right) \geq(1-r)^{1 / 2} \frac{\pi-\arccos (1-2 r)}{\pi}(\pi-s / 2) \\
&>(1-r)^{1 / 2}(1-r)^{1 / 2}(\pi-s / 2) \geq(1-r)(\pi-s / 2) \\
& 8
\end{aligned}
$$


and

$$
|P(\alpha)|=|P(1-2 r)|<T_{2 n}(\sec (s / 4))
$$

follows from Lemma 3.4.

Suppose that (4.7) holds. Then, combining Lemmas 3.5 and 3.7 we obtain

$$
\begin{aligned}
\mu_{[1-2 r, 1]}\left(A_{2}\right) & >r^{1 / 2} \mu_{[-1,1]}\left(A_{2}\right) \geq r^{1 / 2} \frac{\arccos (1-2 r)}{\pi}(\pi-s / 2) \\
& >r^{1 / 2} r^{1 / 2}(\pi-s / 2) \geq r(\pi-s / 2)
\end{aligned}
$$

and

$$
|P(\alpha)|=|P(1-2 r)|<T_{2 n}(\sec (s / 4))
$$

follows from Lemma 3.4*.

Proof of Theorem 2.2. Let $Q \in \mathcal{T}_{n}^{c}(s)$ be odd. Then $R$ defined by $R(t)=2|Q(t)|^{2}-1$ is even and $R \in \mathcal{T}_{2 n}^{c}(s)$. Applying Theorem 2.1 to $R$ we obtain

$$
\left.\max _{t \in K}|2| Q(t)\right|^{2}-1\left|=\max _{t \in K}\right| R(t) \mid \leq \frac{1}{2}\left((\sec (s / 4)+\tan (s / 4))^{4 n}+(\sec (s / 4)-\tan (s / 4))^{4 n}\right),
$$

and hence

$$
\begin{aligned}
\max _{t \in K}|Q(t)| & \leq\left(\frac{1}{4}\left((\sec (s / 4)+\tan (s / 4))^{4 n}+(\sec (s / 4)-\tan (s / 4))^{4 n}\right)+\frac{1}{2}\right)^{1 / 2} \\
& \leq \frac{1}{2}\left((\sec (s / 4)+\tan (s / 4))^{2 n}+(\sec (s / 4)-\tan (s / 4))^{2 n}\right)+\frac{1}{\sqrt{2}}
\end{aligned}
$$

Proof of Theorem 2.3. Observe that if $R \in \mathcal{T}_{n}^{c}(s), s \in(0, \pi)$, and $Q$ is defined by $Q(t)=$ $\frac{1}{2}(R(t)+R(-t))$, then $R \in \mathcal{T}_{n}^{c}(2 s)$. Hence Theorem 2.1 implies that

$$
|R(0)|=|Q(0)| \leq T_{2 n}(\sec (s / 2))
$$

holds for every even $R \in \mathcal{T}_{n}^{c}(s)$ and $s \in(0, \pi)$. The theorem now follows by a simple shift. Namely, observe that $R \in \mathcal{T}_{n}^{c}(s)$ implies that $R_{a} \in \mathcal{T}_{n}^{c}(s)$ where $R_{a}$ is defined by $R_{a}(t):=R(t+a)$ for $t \in K$ and $a \in K$. Hence (4.8) implies that

$$
|R(a)|=\left|R_{a}(0)\right| \leq T_{2 n}(\sec (s / 2))
$$

holds for every even $R \in \mathcal{T}_{n}^{c}(s), s \in(0, \pi)$, and $a \in K$. 


\section{REFERENCES}

1. V. Andrievskii, A note on a Remez-type inequality for trigonometric polynomials, J. Approx. Theory 116 (2002), 416-424.

2. V. Andrievskii Weighted Remez- and Nikolskii-type inequalities on a quasismooth curve, Constr. Approx. (to appear).

3. V. Andrievskii and St. Ruscheweyh, Remez-type inequalities in terms of linear measure, Comput. Methods Funct. Theory 5 (2005), 347-363.

4. B. Bojanov, Elementary proof of the Remez inequality, Amer. Math. Monthly 100 (1993), 483485.

5. P.B. Borwein and T. Erdélyi, Remez-, Nikolskii-, and Markov-type inequalities for generalized nonnegative polynomials with restricted zeros, Constr. Approx. 8 (1992), 343-362.

6. P.B. Borwein and T. Erdélyi, Polynomials and Polynomial Inequalities, Springer-Verlag, New York, N.Y., 1995.

7. P.B. Borwein and T. Erdélyi, Müntz spaces and Remez inequalities, Bull. Amer. Math. Soc. 32 (1995), 38-42.

8. P.B. Borwein and T. Erdélyi, Generalizations of Müntz's Theorem via a Remez-type inequality for Müntz spaces, J. Amer. Math. Soc. 10 (1997), 327 - 349.

9. P.B. Borwein and T. Erdélyi, Pointwise Remez- and Nikolskii-type inequalities for exponential sums, Math. Ann. 316 (2000), 39-60.

10. A. Brudnyi and Yu. Brudnyi, Remez type inequalities and Morrey-Campanato spaces on Ahlfors regular sets, Contemp. Math. 445 (2007), 19-44.

11. A. Brudnyi and Y. Yodmin, Norming sets and related Remez-type inequalities, J. Austral. Math. Soc. 100 (2015), 163-181.

12. R.A. DeVore and G.G. Lorentz, Constructive Approximation, Springer-Verlag, Berlin, 1993.

13. D. Dryanov and Q.I. Rahman, On a polynomial inequality of E.J. Remez, Proc. Amer. Math. Soc. 128 (1999), 1063-1070.

14. T. Erdélyi, The Remez inequality on the size of polynomials, in: Approximation Theory VI, C.K. Chui, L.L. Schumaker, and J.D. Wards, Eds., Academic Press, Boston, 1989, pp. 243-246.

15. T. Erdélyi, A sharp Remez inequality on the size of constrained polynomials, J. Approx. Theory 63 (1990), 335-337.

16. T. Erdélyi, Remez-type inequalities on the size of generalized polynomials, J. London Math. Soc. 45 (1992), 255-264.

17. T. Erdélyi, Remez-type inequalities and their applications, J. Comp. and Applied Math. 47 (1993), 167-210.

18. T. Erdélyi, The Remez inequality for linear combinations of shifted Gaussians, Math. Proc. Cambridge Phil. Soc. 146 (2009), 523-530.

19. T. Erdélyi, X. Li, and E.B. Saff, Remez- and Nikolskii-type inequalities for logarithmic potentials, SIAM J. Math. Anal. 25 (1994), 365-383.

20. T. Erdélyi and P. Nevai, Lower bounds for the derivatives of polynomials and Remez-type inequalities, Trans. Amer. Math. Soc. 349 (1997), 4953-4972.

21. G. Freud, Orthogonal Polynomials, Pergamon Press, Oxford, 1971. 
22. M.I. Ganzburg, Polynomial inequalities on measurable sets and their applications, Constr. Approx. 17 (2001), 275-306.

23. M.I. Ganzburg, Polynomial inequalities on measurable sets and their applications II. Weighted measures, J. Approx. Theory 106 (2000), 77-109.

24. M.I. Ganzburg, On a Remez-type inequality for trigonometric polynomials, J. Approx. Theory 16 (2012), 1233-1237.

25. A. Kroó On Remez-type inequalities for polynomials in $\mathbb{R}^{m}$ and $\mathbb{C}^{m}$, Analysis Mathematica 27 (2001), 55-70.

26. A. Kroó, E.B. Saff, and M. Yattselev, A Remez-type theorem for homogeneous polynomials, J. London Math. Soc. 73 (2006), 783-796.

27. G.G. Lorentz, M. von Golitschek, and Y. Makovoz, Constructive Approximation: Advanced Problems, Springer-Verlag, Berlin, 1996.

28. F. Nazarov, Local estimates for exponential polynomials and their applications to inequalities of the uncertainty principle type, Algebra i Analiz 5 (1993), 3-66.

29. F. Nazarov, Complete version of Turán's lemma for trigonometric polynomials on the unit circumference, in: Complex Analysis, Operators, and Related Topics, The S.A. Vinogradov Memorial Volume, V:P: Havin \& N.K. Nikolskii, Eds., Springer-Verlag, New York, 2000 113, pp. 239-246.

30. E. Nursultanov and S. Tikhonov, A sharp Remez inequality for trigonometric polynomials, Constr. Approx. 38, 101-132.

31. R. Pierzhala, Remez-type inequality on sets with cusps, Adv. Math. 281 (2015), 508-552.

32. E.J. Remez, Sur une propriété des polynômes de Tchebyscheff, Comm. Inst. Sci. Kharkow 13 (1936), 93-95.

33. V. Temlyakov and S. Tikhonov., Remez-type inequalities for the hyperbolic cross polynomials, arXiv:1606.03773 (2016).

34. Y. Yodmin, Remez-type inequality for discrete sets, Israel J. Math. 186 (2011), 45-60.

Department of Mathematics, Texas A\&M University, College Station, Texas 77843, ColLege Station, Texas 77843

E-mail address: terdelyi@math.tamu.edu 